\title{
C DISOCIACIÓN ENTRE LAS EXPERIENCIAS NEGATIVAS Y LA PERCEPCIÓN DE RIESGO DE LAS REDES SOCIALES EN ADOLESCENTES
}

Negative experiences and risk perception
disconnection on the networking sites by teenagers

Belinda De-Frutos-Torres y Mercedes Marcos-Santos

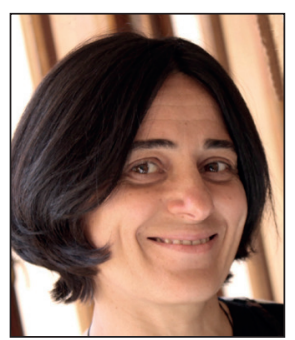

Belinda De-Frutos-Torres es profesora contratada doctora en el Campus de Segovia de la Universidad de Valladolid (España), en la Facultad de Ciencias Sociales, Jurídicas y de la Comunicación. Imparte docencia en la titulación de Publicidad y relaciones públicas. Doctora en psicología por la Universidad Autónoma de Madrid (UAM), llevó a cabo su doctorado en el Departamento de Psicología Social y Metodología de la UAM, en Skidmore University College (New York, EUA) y en Ontario Western University (Canadá). Su trabajo de investigación se centra en la percepción e influencia de la publicidad en internet, y en el uso de las redes sociales por los adolescentes y jóvenes. http://orcid.org/0000-0002-9391-8835

belinda.defrutos@hmca.uva.es

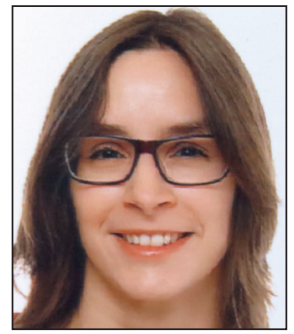

Mercedes Marcos-Santos es licenciada en Publicidad y relaciones públicas y alumna de doctorado de la Universidad de Valladolid en el Campus de Segovia (España). Su línea de investigación principal se centra en la educación y la comunicación a través del análisis del uso de la tecnología e internet y las redes sociales por parte de los menores, así como en la percepción y la influencia de la publicidad online en la formación y desarrollo de adolescentes y jóvenes. http://orcid.org/0000-0002-8567-4172

mmarcos@alumnos.uva.es

Universidad de Valladolid, Facultad de Ciencias Sociales, Jurídicas y de la Comunicación Campus de Segovia Plaza de la Universidad, 1. 40005 Segovia, España

\section{Resumen}

Se presentan los resultados de un estudio en el que se exploró la relación entre la percepción de riesgo en las redes sociales y las experiencias vividas en este entorno. La percepción de seguridad y control sobre los contenidos y la norma social sostienen este tipo de comportamiento entre los adolescentes al margen de las experiencias negativas vividas y la gratificación social recibida. En el estudio participaron 370 alumnos entre 14 y 18 años procedentes de cuatro centros de educación secundaria en Segovia (España). Los resultados muestran que 3 de cada 4 adolescentes han sufrido algún tipo de experiencia negativa en redes sociales, pero estos episodios no influyen en su valoración del riesgo. Se proponen acciones preventivas basadas en la puesta en común de las experiencias negativas en las redes sociales por parte de los adolescentes, y los comportamientos aceptables en ellas.

\section{Palabras clave}

Redes sociales; Adolescentes; Percepción de riesgo; Conductas de riesgo; Gratificación social; Experiencias negativas.

\section{Abstract}

The results of a study exploring the relationship between perception of risk and lived experience by adolescents in online environments are presented. Adolescents reported perceived safety and control over content regardless of negative experiences and social gratification received. The study involved 370 students aged 14 to 18 from four secondary education centers in Segovia (Spain). The results show that three out of four adolescents had encountered some kind of negative experience in social networks, but these episodes did not influence their risk assessment. Preventative actions and acceptable behaviors are proposed for adolescents in social networks.

\section{Keywords}

Social networks; Teenagers; Risky behaviors; Risk perception; Social gratification; Negative social experiences. 
De-Frutos-Torres, Belinda; Marcos-Santos, Mercedes (2017). “Disociación entre las experiencias negativas y la percepción de riesgo de las redes sociales en adolescentes". El profesional de la información, v. 26, n. 1, pp. 88-96.

\section{Introducción}

El acceso a internet y a las redes sociales por parte de los menores es un fenómeno generalizado que ha recibido bastante atención por parte de la investigación aplicada y la comunidad educativa. El $93,6 \%$ de los niños entre 10 y 15 años utiliza internet y el $90,9 \%$ dispone de teléfono móvil; y a partir de los 16 años el 98,3\% accede a internet y posee móvil (INE, 2016). El acceso a las redes y plataformas sociales es una de las actividades que más tiempo ocupan en la esfera interactiva. En España el 87,2\% de los usuarios de internet tiene un perfil activo en las redes sociales (Inteco, 2012), y el $62,0 \%$ de los internautas consultó las redes sociales en el mes de marzo de 2016 según el EGM. Los estudios específicos realizados con adolescentes dan cuenta del alcance que toma el fenómeno de las redes sociales (Bringué; Sádaba, 2009; Hasebrink et al., 2011; Lobe et al., 2011; De-Frutos-Torres; Vázquez-Barrio, 2012; García-Jiménez; López-de-Ayala; Catalina-García, 2013; Bernal; Angulo, 2013; Ólafsson; Livingstone; Haddon, 2014; Sendín-Gutiérrez; Gaona-Pisonero; García-Jiménez, 2014; entre otros).

La rápida incorporación de internet y las redes sociales por parte de chicos y chicas puede ser una fuente de oportunidades para ellos (Livingstone, 2011; Garmendia-Larrañaga et al., 2011; Padilla et al., 2015), al tiempo que genera desconfianza y preocupación entre padres, educadores y la sociedad en general. El uso excesivo de internet, el contacto con personas desconocidas, el acoso que puede recibirse o el acceso a contenidos violentos, racistas o sexuales son riesgos a los que se enfrenta este público vulnerable (Sánchez-Martínez; Otero-Puime, 2010; Garmendia-Larrañaga et al., 2011; Livingstone et al., 2011). Dentro de las redes sociales, el cyberbullying es el riesgo más frecuente. El término recoge el acoso a través de internet, junto con acciones o amenazas mediante mensajes (Slonje; Smith, 2008). Los peligros mencionados con más frecuencia por los menores en las redes sociales se refieren con más frecuencia al comportamiento de sus compañeros que a la relación con extraños. En el discurso de los menores aparecen la agresión verbal repetida, uso no adecuado de información personal, suplantación de identidad, difusión de fotos sin autorización, acoso sexual, habladurías mal intencionadas, daños a la reputación y otras conductas no apropiadas (Livingstone et al., 2013). Buena parte de estos comportamientos no constituyen delito y son acciones ofensivas de baja intensidad.

Recientemente se han emprendido acciones de comunicación para concienciar a los menores sobre los riesgos de internet, si bien no resulta fácil llevar el discurso a la práctica (Jiménez-Iglesias; Garmendia-Larrañaga; Casado-DelRío, 2015). Tales actuaciones preventivas con frecuencia las realizan las fuerzas de seguridad del estado y se centran en acciones delictivas graves, por lo que en general están alejadas de su realidad inmediata y no les capacitan para saber enfrentarse a situaciones problemáticas. A pesar de que los menores son conscientes de los riesgos de internet, la prevención se quiebra en las redes sociales debido a que las perciben como un entorno más seguro que genera desinhibición (Sabater-Fernández, 2014; Martínez-Pastor; SendínGutiérrez; García-Jiménez, 2013). Parece que los menores son conscientes de los comportamientos no apropiados en internet, pero ello no implica que los pongan en práctica en su experiencia diaria en las redes sociales.

Desde la teoría de los usos y gratificaciones (Ruggiero, 2000) se plantea que la búsqueda de información, el entretenimiento y la interacción social son las principales motivaciones para el uso de los medios interactivos, que se convierten en fuentes de gratificación inmediata, particularmente entre los jóvenes (Valkenburg; Soeters, 2001; Bons-Raacke; Raacke, 2008; Dunne; Lawlor; Rowley, 2010). A medida que los niños entran en la adolescencia la faceta social toma mayor relevancia y las redes sociales juegan un papel importante en la construcción de su identidad, su estilo de vida y la relación con sus iguales (Lenhar et al., 2007; Livingstone, 2008; Cáceres-Zapatero; Ruiz-San-Román; Brändle-Señán, 2009; Rubio-Gil, 2010; Ochaita; Espinosa; Gutiérrez, 2011; Colás-Bravo; González-Ramírez; De-Pablos-Pons, 2012).

Por último, la teoría de la acción razonada ${ }^{1}$ (Azjen, 1991) aporta una referencia clave para entender el uso de las redes sociales a través de la norma subjetiva y el control percibido sobre la conducta (Pelling; White, 2009). Los amigos y compañeros son la principal fuente de referencia para el comportamiento. Algunas de las prácticas arriesgadas están normalizadas en el grupo de adolescentes (norma subjetiva). Al estar generalizado el acceso cotidiano a las redes sociales, se va creando confianza y se tiene la sensación de que se controlan ${ }^{2}$ los contenidos, o sea, de que se podrán retirar en cualquier momento y de que nadie va a usarlos indebidamente.

\section{Planteamiento y metodología}

Un primer objetivo de este estudio es identificar la relación entre las experiencias vividas en las redes sociales y la percepción de riesgo en este entorno. Se plantea que las situaciones negativas vividas en las redes sociales contribuirán a una mayor percepción de riesgo, mientras que las experiencias gratificantes derivadas del entretenimiento, la información y la interacción social lo contrarrestarán. Por otra parte, el riesgo está relacionado con el control: cuanto mayor sea la sensación de seguridad menor será el riesgo percibido.

Aunque no hay trabajos específicos sobre la percepción de riesgo en las redes sociales sí se han identificado determinados factores a tener en cuenta:

- frecuencia de uso de las plataformas de redes sociales (Del-Río; Sádaba; Bringué, 2010; Hasebrink et al., 2011; Staksrud; Ólafsson; Livingstone, 2013; García-Jiménez; 
Catalina-García; Montes-Vozmediano, 2015);

- privacidad y tipo de información mostrado en los perfiles (Sengupta; Chaudhuri, 2011; Staksrud; Ólafsson; Livingstone, 2013);

género (Catalina-García; López-de-Ayala; García-Jiménez, 2014; Kormas et al., 2011; Durkee et al., 2012; Rial et al., 2014; Catalina-García; López-de-Ayala; GarcíaJiménez, 2014; Alfaro-González et al., 2015; Fernández; Peñalva; Irazábal, 2015); y

- diferencias individuales en el patrón de uso de internet (Hasebrink et al., 2011; De-Frutos-Torres; Sánchez-Valle; Vázquez-Barrio, 2014).

Teniendo en cuenta estos resultados se plantea un segundo objetivo centrado en identificar el perfil de las conductas de riesgo a partir de las variables personales, conductuales y actitudinales que puedan estar asociadas con prácticas poco seguras en las redes sociales.

Los menores están familiarizados con

los riesgos de las redes sociales, pero ello no se traduce necesariamente en un comportamiento preventivo en ellas

\subsection{Muestra}

El estudio se llevó a cabo con una muestra de 370 participantes seleccionados en centros de educación secundaria ubicados en Segovia (España). Se seleccionaron al azar dos centros públicos y dos centros privados-concertados ${ }^{3}$ y se eligieron alumnos de tercer y cuarto curso de Educación Secundaria Obligatoria (ESO). Se solicitó la colaboración en el estudio a los directores de los centros a través de la Fundación $A N A R$, los participantes fueron informados del anonimato y de la confidencialidad de los datos recogidos, y su participación fue voluntaria. La muestra estuvo integrada en un $53 \%$ por chicos y un $47 \%$ por chicas; el $60 \%$ pertenece al tercer curso de ESO y el otro $40 \%$ al cuarto. El rango de edad se situaba entre los 14 y 18 años, con una media de 15,3 años (desviación típica $=1$ ).

\subsection{Procedimiento}

Se diseñó un cuestionario, que fue cumplimentado en el aula con la presencia de una persona del equipo de investigación, e incluía los datos personales: edad, sexo y curso académico. Las variables sobre redes sociales incluidas en el estudio se detallan a continuación:

\section{Uso}

- tiempo de conexión, que se identificó marcando los períodos de conexión en ocho franjas horarias a lo largo del día;

- número de cuentas abiertas; y

- nombre de las redes utilizadas habitualmente.

\section{Acciones poco seguras}

- contactar con desconocidos;

- mantener el perfil público o parcialmente público; e

- incluir en el perfil la dirección, el teléfono o el colegio.

\section{Incidentes negativos}

- agresiones;

- contenidos molestos;

- difusión de rumores sobre el propio individuo;

- difusión de rumores de otras personas, y

- otros comportamientos inapropiados, que aunque no lleguen a ser una falta o un delito constituyen experiencias negativas.

\section{Gratificaciones por usar las redes sociales}

- tener muchos amigos;

- hacer comentarios sobre los contenidos que suben los amigos;

- mostrar una buena imagen de uno mismo; y

- subir contenidos interesantes.

Las respuestas se recogieron en una escala tipo Likert de 5 puntos, de nada a muy importante. La puntuación global se obtuvo con la media de las puntuaciones de los items de la escala. La consistencia interna de la escala medida con el coeficiente alpha de Cronbach fue de 0,65.

El estilo de navegación se evaluó con la escala de De-FrutosTorres; Sánchez-Valle; Vázquez-Barrio (2014): una puntuación alta implica una conducta experimental en la navegación que busca entretenimiento, y estimulación, y supone comportamientos arriesgados. La escala, formada por seis posiciones, obtiene una puntuación media de 3,0 puntos $(S D=0,56)$ y un coeficiente de fiabilidad alpha de Cronbach de 0,67 .

Las percepciones de control sobre los contenidos subidos a la red social, y sobre la seguridad en las redes sociales se recogieron en una escala Likert de 5 puntos.

Los jóvenes perciben las redes sociales como medios gratificantes por el valor de entretenimiento e interacción social, además del lugar donde expresar la propia identidad

Finalmente, se pidió a los participantes que valoraran hasta qué punto consideraban que las redes sociales podrían llevarles a situaciones de riesgo, las respuestas se recogieron en una escala Likert de 5 puntos, donde 1 indicaba un alto riesgo y 5 ausencia de riesgo. Las puntuaciones de las variables se han recodificado de manera que mayor puntuación implica mayor percepción de riesgo.

\section{Resultados y discusión}

\subsection{Acceso a las redes sociales}

El 4,3\% de los participantes indicó que no tenía cuenta abierta en ninguna red social. Dado que el estudio se centra en el uso de las redes sociales los resultados se refieren a la muestra válida formada por $\mathbf{3 5 4}$ menores que sí las usaban. Los adolescentes suelen utilizar más de una cuenta: el $49,2 \%$ tiene entre dos y tres cuentas, otro $20,3 \%$ declara 4 


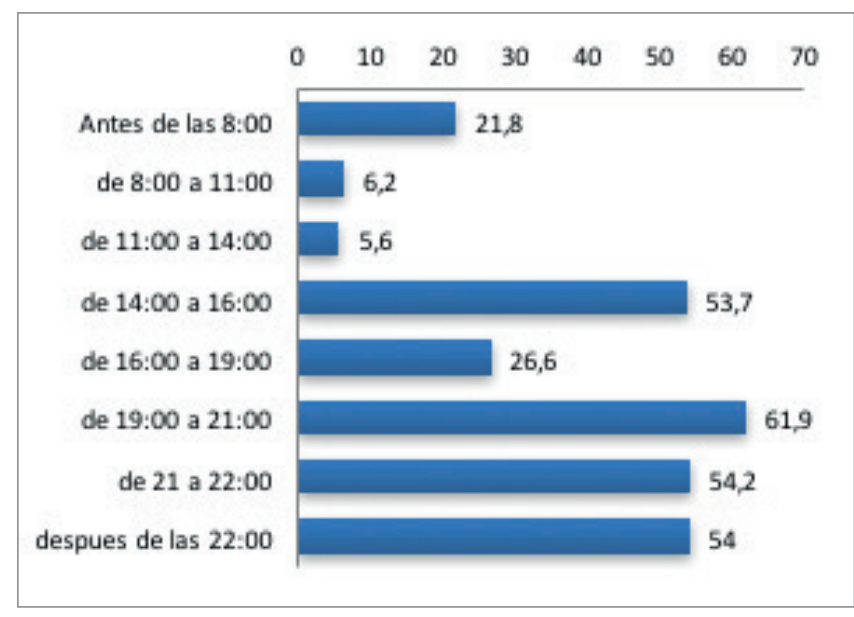

Gráfico 1. Conexión a las redes sociales a lo largo de un día laborable (\%)

cuentas activas y el 18,9\% está suscrito a 5 ó más cuentas. Tener una sola cuenta ha resultado ser lo menos frecuente $(7,3 \%)$. Las horas de la tarde, particularmente entre las 19:00 y 21:00 horas, son las preferidas para conectarse a las redes sociales según se muestra en el gráfico 1 . No obstante la conexión sigue por la noche para más de la mitad de los participantes. Ante la dificultad de medir el tiempo global de consumo se ha hecho una estimación sumando los períodos marcados por cada participante. La mitad se conecta en 3 de los períodos (media $=2,89$ y deviación típica 1,59) que equivale aproximadamente a unas 9 horas al día (gráfico 1 ).

\subsection{Conductas de riesgo}

El $66,1 \%$ afirma tener su perfil privado para que sólo puedan verlo sus amigos, ésta se considera una práctica segura. El resto declara prácticas más arriesgadas, en concreto, un $17,5 \%$ tiene parcialmente privado su perfil (pueden verlo amigos de amigos) y un $16,4 \%$ de los casos está abierto a cualquier persona. El porcentaje de perfiles seguros es superior al recogido en el estudio EUkids online (Lobe et al., 2011). Además, se considera una conducta poco segura proporcionar datos personales como la dirección, el número de teléfono y el centro escolar. El 7,3\% de los casos incluye la dirección, el $17,8 \%$ el número de teléfono y el $51,1 \%$ el cen-

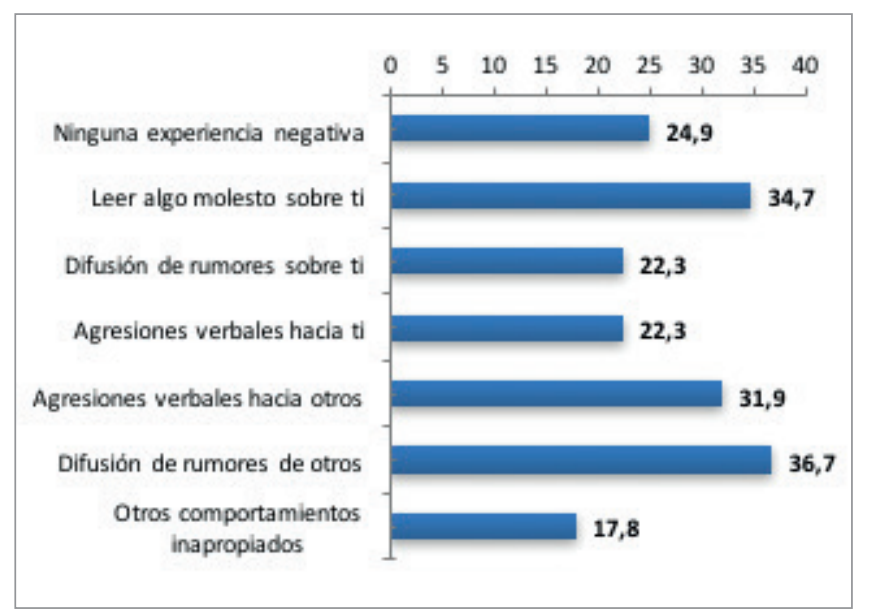

Gráfico 3. Incidencia de experiencias negativas en las redes sociales (\%)

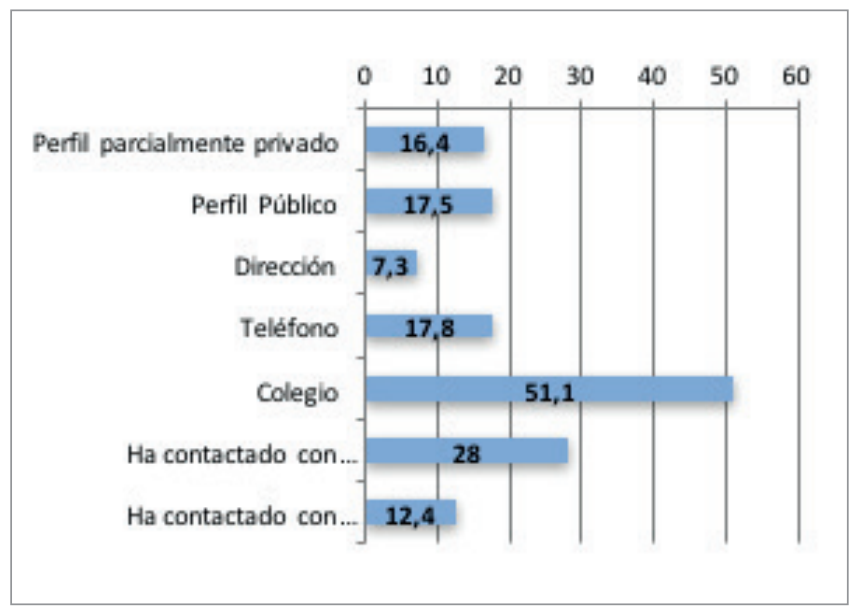

Gráfico 2. Incidencia de las conductas de riesgo (\%)

tro de estudios. Aunque más de la mitad de los participantes nunca ha entrado en contacto con desconocidos, un $28 \%$ informa de que alguna vez lo ha hecho pero no tiene intenciones de volverlo a repetir y un $12,4 \%$ no ve inconveniente en reincidir sobre esta conducta (gráfico 2).

En la experiencia diaria de las redes sociales los jóvenes se enfrentan con situaciones desagradables. Aunque la frecuencia con la que se relatan este tipo de hechos fluctúa entre el $17,8 \%$ y el $34,7 \%$, son acciones que se dan simultánea y repetidamente. Sólo uno de cada cuatro participantes declara que no ha tenido ninguna experiencia negativa en su uso de las redes sociales, proporción algo mayor de la recogida en otros estudio similares (Sabater-Fernández; López-Hernáez, 2015) (gráfico 3).

Por otra parte, las redes sociales constituyen una fuente de gratificación gracias a la interacción social, la aceptación y la imagen mostrada ante los demás. La puntuación media de la escala es de 3,3 puntos y la desviación típica es igual a 0,55 (tabla 1).

La opinión de los adolescentes sobre el control de los contenidos subidos a las redes es bastante optimista: su valoración media está en 3,82 puntos sobre una escala de cinco puntos. En este mismo sentido apunta la variable sobre la seguridad en las redes sociales, la confianza es algo mayor al evaluar la seguridad en las redes sociales. Finalmente, la

Tabla 1. Respuestas a la evaluación de control de contenidos, seguridad y percepción de riesgos en las redes sociales, puntuación media y desviación típica

\begin{tabular}{|c|c|c|c|c|c|c|c|}
\hline & \multicolumn{3}{|c|}{$\begin{array}{l}\text { Ausencia } \\
\text { (control, } \\
\text { seguridad, } \\
\text { riesgo) }\end{array}$} & \multicolumn{2}{|c|}{$\begin{array}{r}\text { Totalmente } \\
\text { (controlado, } \\
\text { seguro, arries- } \\
\text { gado) }\end{array}$} & \multirow[t]{2}{*}{$\begin{array}{l}\text { Me- } \\
\text { dia }\end{array}$} & \multirow[t]{2}{*}{$\begin{array}{c}\text { Des- } \\
\text { viación } \\
\text { típica }\end{array}$} \\
\hline & 1 & 2 & 3 & 4 & 5 & & \\
\hline $\begin{array}{l}\text { Control de } \\
\text { contenidos }\end{array}$ & $4,8 \%$ & $6,8 \%$ & $23,4 \%$ & $31,9 \%$ & $33,1 \%$ & 3,82 & $(1,11)$ \\
\hline Seguridad & $1,1 \%$ & $7,1 \%$ & $18,9 \%$ & $41,2 \%$ & $31,6 \%$ & 3,95 & $(0,94)$ \\
\hline $\begin{array}{l}\text { Riesgo } \\
\text { percibido }\end{array}$ & $9,3 \%$ & $16,9 \%$ & $40,4 \%$ & $21,5 \%$ & $11,9 \%$ & 3,10 & $(1,11)$ \\
\hline
\end{tabular}


percepción de riesgo se sitúa en medio de la escala con una puntuación de 3,10 (desviación típica $=1,1$ ) en la escala de 5 puntos, que se puede interpretar como un equilibrio entre el peligro y la seguridad.

\subsection{Perfil asociado a las prácticas de riesgo en las re- des sociales}

Para identificar las variables que puedan tener relación con las conductas de riesgo se ha efectuado un análisis de regresión logística. A los participantes que habían señalado alguno de los comportamientos poco seguros se les ha asignado al grupo de riesgo (código 1). El análisis se ha efectuado por el método de pasos sucesivos para identificar las variables con mayor poder predictivo sobre la variable dependiente. Las variables incluidas en el análisis han sido la edad, el sexo, el tiempo de conexión a las redes sociales, el número de cuentas abiertas, el estilo de navegación exploratorio, la gratificación social y las experiencias negativas vividas en las redes sociales. El modelo es significativo (Chi-cuadrado $=6,911$ sig. $=0,009$ ).

\section{Las chicas adolescentes valoran más que} los chicos el riesgo en las redes sociales

Los resultados indican que la variable más relacionada con las conductas de riesgo es el estilo de navegación exploratorio. La razón de la ventaja es de 2,8 (Exp (b) =2,8), es decir, cuanto más internalizada está la navegación exploratoria mayor es la probabilidad de incurrir en conductas de riesgo en las redes sociales. En segundo lugar entra en la ecuación de regresión el número de cuentas abiertas en las redes sociales, la razón de la ventaja para esta variable es 1,32: a medida que se incrementa el número de cuentas abiertas mayor es la probabilidad de tener conductas arriesgadas. La gratificación social recibida a través de las redes sociales es la siguiente variable que está asociada con las conductas de riesgo. En este caso la razón de las ventajas también es mayor que 1, por lo tanto, a medida que se le da más importancia a las redes sociales como fuente de gratificación, mayor es la probabilidad de implementar comportamientos arriesgados. Las tres variables incluidas en la ecuación de regresión logística explican un $11,8 \%$ de las conductas de riesgo ( $R^{2}$ de Nagelkerke $\left.=0,118\right)$. El porcentaje de acierto en el pronóstico sobre las conductas de riesgo es del $80,2 \%$.

El análisis de las variables que no entran en la ecuación de regresión logística sirven también de apoyo para la discusión de los resultados. Ni la edad, ni el sexo están asociadas

Tabla 2. Análisis de regresión logística sobre las conductas de riesgo, método de pasos sucesivos

\begin{tabular}{|l|c|c|c|}
\cline { 2 - 4 } \multicolumn{1}{c|}{} & Coeficiente Beta (ET) & Wald (sig) & Exp (B) \\
\hline Constante & $-3,604(1,097)$ & $-3,438(0,001)$ & 0,027 \\
\hline $\begin{array}{l}\text { Estilo de navega- } \\
\text { ción exploratorio }\end{array}$ & $0,737(0,297)$ & $6,143(0,013)$ & 2,090 \\
\hline $\begin{array}{l}\text { No de cuentas } \\
\text { abiertas }\end{array}$ & $0,276(0,110)$ & $6,279(0,012)$ & 1,318 \\
\hline Gratificación & $0,642(0,277)$ & $5,064(0,024)$ & 1,866 \\
\hline
\end{tabular}

con las conductas de riesgo, a diferencia de los estudios referenciados donde sí se habían encontrado diferencias en base a variables personales. Así mismo resulta revelador que mientras la gratificación social que proporcionan las redes a los adolescentes está vinculada con las prácticas de riesgo, haber vivido experiencias negativas no lo está.

\section{A pesar de haber vivido experiencias ne- gativas en las redes sociales, los menores no las perciben como sitios con riesgo}

\subsection{Factores asociados al riesgo percibido en las re- des sociales}

A continuación se buscan los factores asociados al riesgo percibido en la redes sociales. Se plantea que el riesgo depende del control de la situación, en este caso, el control sobre los contenidos subidos a las redes sociales, y la seguridad en las redes sociales sirven de referencia para el análisis. Además se propone que las experiencias acumuladas en las redes sociales, tanto positivas como negativas, serán un factor de influencia en la percepción de riesgo, además de las variables personales (edad, sexo, estilo de navegación) y de las variables de uso (cuentas abiertas y tiempo de conexión). Para poner a prueba este modelo de influencia se ha llevado a cabo un análisis de regresión lineal sobre la percepción de riesgo en dos bloques. En el primer bloque se han introducido las variables sexo, edad, número de perfiles de redes sociales y frecuencia de conexión. El análisis de la regresión es estadísticamente significativo (Anova $\mathrm{F}=2,508$; $4 / 349$; sig. $=0,042$ ) y explica el $2,8 \%$ de la percepción del riesgo $\left(R^{2}=0,028\right)$. En el segundo bloque se incluyen el resto de variables, lo que supone una mejora significativa del modelo (Durbin Watson=2,157; sig. 0,009), y un incremento de la varianza explicada hasta el 7,9\% $\left(R^{2}=0,079\right)$.

En la tabla 3 se muestra la estimación de los parámetros de la ecuación de regresión. Sobre las relaciones propuestas sólo

Tabla 3. Análisis de regresión sobre la percepción de riesgo. Método de introducción de variables

\begin{tabular}{|l|l|l|l|}
\cline { 2 - 4 } \multicolumn{1}{c|}{} & Coeficiente Beta & \multicolumn{1}{c|}{ t } & sig. \\
\hline Bloque 1 & B (error típico) & & \\
\hline Constante & $2,383(0,915)$ & 2,603 & 0,010 \\
\hline Edad & $0,007(0,059)$ & 0,112 & 0,911 \\
\hline Sexo & $0,279(0,118)$ & $\mathbf{2 , 3 6 3}$ & 0,019 \\
\hline No de cuentas abiertas & $0,080(0,044)$ & 1,838 & 0,067 \\
\hline Tiempo de conexión diaria & $-0,023(0,039)$ & $-0,586$ & 0,558 \\
\hline
\end{tabular}

\begin{tabular}{|l|l|l|l|}
\hline Bloque 2 & & & \\
\hline Control sobre los contenidos & $-0,154(0,058)$ & $\mathbf{- 2 , 6 6 4}$ & 0,008 \\
\hline Seguridad percibida & $-0,066(0,069)$ & $-0,957$ & 0,339 \\
\hline Estilo de navegación exploratorio & $0,101(0,121)$ & 0,833 & 0,406 \\
\hline Conductas de riesgo & $0,000(0,036)$ & 0,013 & 0,990 \\
\hline Experiencias negativas & $-0,024(0,038)$ & $-0,631$ & 0,529 \\
\hline Gratificación & $-0,032(0,103)$ & $-0,312$ & 0,755 \\
\hline
\end{tabular}


dos variables obtienen parámetros estadísticamente significativos. En el bloque de las variables personales el sexo tiene un efecto sobre la percepción del riesgo, las chicas adolescentes realizan una valoración de los riesgos más elevada. El control sobre los contenidos subidos a la red está asociado con la percepción de riesgo y el sentido de la relación es negativo, es decir, cuanto mayor es la sensación de que nadie usa indebidamente los contenidos propios menor es el riesgo percibido en las redes sociales. Quizá el aspecto más revelador de este análisis sea la ausencia de relación del riesgo percibido con las experiencias vividas por los menores en las redes sociales, ya sean de signo negativo como aquellas que son fuente de gratificación de las redes, y la ausencia de relación con las conductas de riesgo en las redes sociales.

\section{El control sobre los contenidos subidos} a la Red se asocia inversamente con la percepción de riesgo: cuanto mayor es la sensación de control sobre los contenidos compartidos en las redes sociales menor es el riesgo percibido

\section{Conclusiones}

La principal conclusión es la disociación entre la percepción del riesgo y las experiencias en las redes sociales. Cabría esperar que las experiencias negativas fueran un estímulo para que los menores sean más conscientes de los riesgos a los que pueden estar expuestos en las redes sociales. Sin embargo los resultados muestran que no hay relación entre ambos aspectos. Del mismo modo, las experiencias positivas derivadas de las redes sociales no se traducen en una disminución del riesgo percibido. Tan sólo el género ha mostrado tener un papel significativo en la percepción de riesgo de las redes sociales que es mayor en las chicas adolescentes.

Este resultado explica la dificultad para concienciar sobre los riesgos si ni siquiera la propia experiencia sirve de referencia para tomar conciencia de las situaciones difíciles a las que les pueden llevar las redes sociales. Se podría pensar que los menores que han participado en el estudio no han vivido malas experiencias y por eso resulta difícil tomar conciencia del peligro. Los resultados muestran que tres de cada cuatro menores había sufrido algún tipo de incidente desagradable en su experiencia de uso. Por otra parte, es evidente que las redes sociales juegan un rol muy importante en su vida. La cuestión es cómo conciliar ambas cuestiones. Desde nuestro punto de vista la disonancia cognitiva podría explicar esta aparente disociación. Las redes sociales son una fuente de gratificación social para los menores, que sostienen comportamientos arriesgados, que a su vez pueden tener consecuencias negativas. Para evitar las tensiones que pueda generar esta contradicción los menores pueden negar las consecuencias aumentando su sensación de control sobre las redes.

A partir del estudio se recomienda que las acciones de prevención de riesgos en las redes sociales tengan en cuenta la aparente contradicción. Sería interesante fomentar la discusión y puesta en común por los propios adolescentes sobre las experiencias negativas que viven con frecuencia en las redes sociales, concienciar sobre la importancia de cuestionar dentro del propio grupo comportamientos que no sean aceptables. De este modo se podrían evitar los efectos negativos de su normalización, contribuiría a tener una noción más realista de los riesgos que con más frecuencia se viven en las redes sociales y se fomentaría el control sobre los contenidos.

Finalmente el estudio demuestra la relación de determinados comportamientos arriesgados con determinadas variables personales y actitudinales. No sorprende ver que los menores que utilizan las redes sociales con mayor frecuencia tienen más probabilidad de incurrir en conductas de riesgo y de sufrir experiencias negativas. El número de cuentas abiertas es otra variable que está asociada al riesgo. $\mathrm{Ni}$ la edad, ni el sexo están vinculadas al comportamiento arriesgado, pero sí encontramos una variable actitudinal respecto a la interacción con internet, que es la navegación exploratoria. La mayor predisposición a la búsqueda de nuevas experiencias, contenidos, etc., en la Red lleva a una mayor exposición a los riesgos.

\section{Notas}

1. La teoría de la acción razonada plantea que las personas configuran las creencias y actitudes sobre su propia conducta teniendo en cuenta la opinión de los demás (norma subjetiva) y el control sobre la misma (control percibido de la conducta), contribuyendo en su conjunto a predecir el comportamiento. La norma subjetiva refleja la presión social percibida por el individuo para llevar a cabo esa conducta. El control percibido de la conducta recoge la facilidad o dificultad para implementar la acción.

2. Subir contenidos a la Red es un acto voluntario, bajo nuestro control, pero en el momento que se comparte se pierde parte del control. Nos referimos a "control" en el sentido de que si hoy se sube una imagen y mañana se decide eliminarla se pueda hacer, que se pueda decidir quién la ve, y en qué momento, etc.

El uso frecuente de las redes establece la creencia de que se está en un entorno que controlado, cuando en realidad, si se sube un contenido y se comparte se deja de tener control sobre ese contenido, y otras personas pueden seguir compartiéndolo entre sus contactos.

El control depende del nivel de privacidad establecido en los perfiles, pero aun así, compartir información entre "amigos", puede llevar a situaciones poco deseadas. Es frecuente entre parejas de adolescentes compartir fotos íntimas; si más tarde se rompe la relación, una de las partes puede utilizar estas fotos para deteriorar la imagen del otro haciéndolas públicas. En ese sentido, se pierde el control de la información. Se tiende a creer que se tiene el control sobre el propio perfil, pero es una idea ilusoria, porque realmente la seguridad se puede romper fácilmente.

3. Segovia cuenta con 6 centros de formación secundaria públicos ubicados en la capital, con características socioeconómicas similares y 3 centros privados-concertados. Por ello la elección de los centros se realizó por un procedimiento aleatorio simple. 


\section{Anexo}

Correlación de Pearson de las variables utilizadas en el análisis de regresión

\begin{tabular}{|c|c|c|c|c|c|c|c|c|c|}
\hline & $\begin{array}{c}\text { Control } \\
\text { sobre los } \\
\text { contenidos }\end{array}$ & Seguridad & $\begin{array}{c}\text { Expe- } \\
\text { riencias } \\
\text { negativas }\end{array}$ & $\begin{array}{l}\text { Gratifica- } \\
\text { ción social }\end{array}$ & $\begin{array}{c}\text { Estilo de } \\
\text { navegación } \\
\text { exploratorio }\end{array}$ & $\begin{array}{l}\text { Períodos de } \\
\text { consulta }\end{array}$ & $\begin{array}{l}\text { Cuentas } \\
\text { abiertas }\end{array}$ & Sexo & Edad \\
\hline Percepción de riesgo & $-0,163^{* *}$ & $-0,107^{*}$ & $-0,022$ & 0,004 & 0,032 & 0,018 & $0,109^{*}$ & $0,136^{*}$ & 0,010 \\
\hline $\begin{array}{l}\text { Control sobre los } \\
\text { contenidos }\end{array}$ & & $0,437^{* *}$ & $-0,007$ & 0,034 & 0,025 & 0,045 & 0,064 & 0,068 & $-0,010$ \\
\hline Seguridad & & & 0,104 & $0,111^{*}$ & 0,080 & 0,053 & $0,136^{*}$ & 0,037 & 0,005 \\
\hline Experiencias negativas & & & & $0,125^{*}$ & 0,078 & $0,223^{* *}$ & $0,137^{* *}$ & 0,044 & $0,113^{*}$ \\
\hline Gratificación social & & & & & $0,335^{* *}$ & $0,177^{* *}$ & $0,132^{*}$ & $0,113^{*}$ & $-0,039$ \\
\hline $\begin{array}{l}\text { Estilo de navegación } \\
\text { exploratorio }\end{array}$ & & & & & & $0,022^{* *}$ & $0,261^{* *}$ & $-0,168^{* *}$ & $0,140^{* *}$ \\
\hline Períodos consulta & & & & & & & & 0,070 & $0,184^{* *}$ \\
\hline Cuentas abiertas & & & & & & & & $0,125^{*}$ & 0,055 \\
\hline Sexo & & & & & & & & & 0,020 \\
\hline Edad & & & & & & & & & \\
\hline
\end{tabular}

$\mathrm{p}<0,05 * * \mathrm{p}<0,005$

\section{Agradecimientos}

Las autoras quieren agradecer a la fundación $A N A R$ y los centros de educación secundaria colaboradores del estudio su participación desinteresada. A los revisores anónimos de este trabajo por sus sugerencias y mejoras del texto. Este trabajo de investigación se enmarca en el proyecto de investigación Competencias mediáticas de la ciudadanía en medios digitales emergentes en el ámbito profesional de la comunicación, financiado por MIMECO/FEDER Ref. EDU2015-64015-C3-3-R.

\section{Referencias}

Ajzen, Icek (1991). "The theory of planned behavior". Organizational behavior and human decision processes, v. 50, pp. 179-211.

https://doi.org/10.1016/0749-5978(91)90020-T

Alfaro-González, María; Vázquez-Fernández, Marta-Esther; Fierro-Urturi, Ana; Herrero-Bregón, Beatriz; Muñoz-Moreno, M. Fátima; Rodríguez-Molinero, Luis (2015). “Uso y riesgos de las tecnologías de la información y comunicación en adolescentes de 13-18 años". Acta pediátrica española, v. 73, n. 6 , pp. 146-151.

http://www.actapediatrica.com/index.php/secciones/ originales/1127-uso-y-riesgos-de-las-tecnologias-de-lainformacion-y-comunicacion-en-adolescentes-de-13-18-anos

Bernal, César; Angulo, Félix (2013). “Interacciones de los jóvenes andaluces en las redes sociales". Comunicar, v. 20, n. 40, pp. $25-30$.

https://doi.org/10.3916/C40-2013-02-02

Bonds-Raacke, Jennifer; Raacke, John (2008). "MySpace and Facebook: Applying the uses and gratifications theory to exploring friend-networking sites". Cyberpsychology \& behavior, v. 8, n. 1, pp. 27-33.

https://doi.org/10.1089/cpb.2007.0056
Bringué, Xavier; Sádaba, Charo (2009). La generación interactiva en España. Niños y adolescentes ante las pantallas. Barcelona: Ariel, Colección Fundación Telefónica. ISBN: 978 8408091110

Cáceres-Zapatero, María-Dolores; Ruiz-San-Román, José A.; Brändle-Señán, Gaspar (2009). Comunicación interpersonal y vida cotidiana. La presentación de la identidad de los jóvenes en internet. CIC: Cuadernos de información y comunicación, n. 14, pp. 213-231.

http://revistas.ucm.es/index.php/CIYC/article/view/8070

Catalina-García, Beatriz; López-de-Ayala, María-Cruz; García-Jiménez, Antonio (2014). "Los riesgos de los adolescentes en internet: los menores como actores y víctimas de los peligros en internet". Revista latina de comunicación social, n. 69, pp. 462-485.

https://doi.org/10.4185/RLCS-2014-1020

Colás-Bravo, Pilar; González-Ramírez, Teresa; De-PablosPons, Juan (2013). "Juventud y redes sociales: motivaciones y usos preferentes". Comunicar, v. 20, n. 40, pp. 15-23. https://doi.org/10.3916/C40-2013-02-01

De-Frutos-Torres, Belinda; Sánchez-Valle, María; VázquezBarrio, Tamara (2014). "Perfiles de adolescentes on line y su comportamiento en el medio interactivo". Icono 14, v. 12, n. 1, pp. 374-397.

https://doi.org/10.7195/ri14.v11i2.208

De-Frutos-Torres, Belinda; Vázquez-Barrio, Tamara (2012). "Adolescentes y jóvenes en el entorno digital: análisis de su discurso sobre usos, percepción de riesgo y mecanismos de protección". Doxa comunicación, n. 15, pp. 57-79.

http://www.doxacomunicacion.es/es/hemeroteca/ articulos?id=135

Del-Río, Jorge; Sádaba, Charo; Bringué, Xavier (2010). “Menores y redes ¿sociales?: de la amistad al cyberbu- 
llying". Juventud y nuevos medios de comunicación, n. 88, pp. 115-129.

http://www.injuve.es/sites/default/files/RJ88-09.pdf

Dunne, Aine; Lawlor, Margaret Anne; Rowley, Jennifer (2010). "Young people's use of online social networking sites-a uses and gratifications perspective". Journal of research in interactive marketing, v. 4, n. 1, pp. 46-58. https://doi.org/10.1108/17505931011033551

EGM (Estudio general de medios) (2016). Audiencia de Internet en el EGM (abril-mayo 2016).

http://www.aimc.es

Durkee, Tony; Kaess, Michael; Carli, Vladimir; Parzer, Peter; Wasserman, Camilla; Floderus, Birgitta; Apter, Alan; Balazs, Judit., Barzilay, Shira; Bobes, Julio; Brunner, Romuald; Corcoran, Paul; Cosman, Doina; Cotter, Padraig; Despalins, Romain; Graber, Nadja; Guillemin, Francis; Haring, Christian; Kahn, Jean-Pierre; Mandelli, Laura, Marusic, Dragan; Mészáros; Gergely, Musa, George J.; Postuvan, Vita; Resch, Franz; Saiz, Pilar A.; Sisask, Merike; Varnik, Airi; Sarchiapone, Marco; Hoven, Christina W.; Wasserman, Danuta (2012). "Prevalence of pathological internet use among adolescents in Europe: Demographic and social factors". Addiction, v. 107, n. 12, pp. 2210-2222.

https://doi.org/10.1111/j.1360-0443.2012.03946.x

http://onlinelibrary.wiley.com/enhanced/exportCitation/ doi/10.1111/j.1360-0443.2012.03946.x

Fernández, Javier; Peñalva, María Alicia; Irazábal, Itziar (2015). "Hábitos de uso y conductas de riesgo en internet en la pre-adolescencia". Comunicar, v. 22, n. 44, pp. 113-120. https://doi.org/10.3916/C44-2015-12

García-Jiménez, Antonio; Catalina-García, Beatriz; MontesVozmediano, Manuel (2015). “Una aproximación a la educación mediática para el contacto con desconocidos en internet". Comunicación y hombre, n. 11, pp. 101-114.

http://ddfv.ufv.es/xmlui/handle/10641/1183

http://hdl.handle.net/10641/1183

García-Jiménez, Antonio; López de Ayala, María-Cruz; Catalina-García, Beatriz (2013). "Hábitos de uso en internet y en las redes sociales de los adolescentes españoles". Comunicar, v. 21, n. 41, pp. 195-204.

https://doi.org/10.3916/C41-2013-19

Garmendia-Larrañaga, Maialen; Garitaonandia-Garnacho, Carmelo; Martínez-Fernández, Gemma; Casado-Del-Río, Miguel-Ángel (2011). Riesgos y seguridad en internet. Los menores españoles en el contexto europeo. Bilbao: Universidad del País Vasco/Euskal Herriko Unibertsitatea, EU Kids Online. ISBN: 9788498605204

Hasebrink, Uwe; Görzig, Anke; Haddon, Leslie; Kalmus, Veronika; Livingstone, Sonia (2011). Patterns of risk and safety online: In-depth analyses from the EU Kids Online survey of 9-to 16-year-olds and their parents in 25 European countries. EU kids online, deliverable D6. EU Kids Online Network, London, UK.

http://eprints.Ise.ac.uk/39356/1/Patterns_of_risk_and_ safety_online_(LSERO).pdf

INE (Instituto Nacional de Estadística) (2015). Encuesta so- bre equipamiento y uso de tecnologías de información y comunicación en los hogares. Año 2015.

http://www.ine.es/dyngs/INEbase/es/operacion.htm?c= Estadistica_C\&cid=1254736176741\&menu=ultiDatos\&i $d p=1254735976608$

Jiménez-Albiar, María-Isabel; Piqueras-Rodríguez, JoséAntonio; Mateu-Martínez, Ornela; Carballo, José-Luis; Orgilés-Amorós, Mireia; Espada-Sánchez, José-Pedro (2012). "Diferencias de sexo, característica de personalidad y afrontamiento en el uso de internet, el móvil y los videojuegos en la adolescencia". Health and addictions / Salud y drogas, v. 12, n. 1, pp. 61-82.

http://ojs.haaj.org/index.php/haaj/article/view/10/10

Jiménez-Iglesias, Estefanía; Garmendia-Larrañaga, Maialen; Casado-Del-Río, Miguel-Ángel (2015). “Percepción de los y las menores de la mediación parental respecto a los riesgos en internet". Revista latina de comunicación social, n. 70, pp. 49-68.

http://www.revistalatinacs.org/070/paper/1034-UP/04es.html

Kormas, Georgios; Critselis, Elena; Janikian, Mari; Kafetzis, Dimitrios; Tsitsika, Artemis (2011). "Risk factors and psychosocial characteristics of potential problematic and problematic internet use among adolescents: A cross-sectional study". BMC Public health, v. 11, n. 1, pp. 595-602.

https://doi.org/10.1186/1471-2458-11-595

Lenhart, Amanda; Madden, Mary; MacGill, AlexandraRankin; Smith, Aaron (2007). "Teens and social media: The use of social media gains a greater foothold in teen life as they embrace the conversational nature of interactive online media". PEW Internet \& American Life Project, 44 pp. http://www.pewinternet.org/files/old-media/Files/ Reports/2007/PIP_Teens_Social_Media_Final.pdf.pdf

Livingstone, Sonia (2008). "Taking risky opportunities in youthful content creation: Teenagers' use of social networking sites for intimacy, privacy and self-expression". New media \& society, v. 10, n. 3, pp. 393-411.

https://doi.org/10.1177/1461444808089415

Livingstone, Sonia; Haddon, Leslie; Görzig, Anke; Ólafsson, Kjartan (2011). Technical report and user guide: The 2010 EU kids online survey. EU Kids Online, London School of Economics and Political Science, London.

http://www.Ise.ac.uk/media@lse/research/EUKidsOnline/ EU\%20Kids\%2011\%20(2009-11)/Survey/Technical\%20 report.pdf

Livingstone, Sonia; Helsper, Ellen J. (2007). "Taking risks when communicating on the internet: The role of offline social-psychological factors in young people's vulnerability to online risks". Information, Communication \& society, v. 10 , n. 5, pp. 619-644.

https://doi.org/10.1080/13691180701657998

Livingstone, Sonia; Kirwil, Lucyna; Ponte, Cristina; Staksrud, Elisabeth (2013). In their own words: What bothers children online? EU Kids Online Network. LSE London: EU Kids On-line.

http://www.Ise.ac.uk/media@Ise/research/EUKidsOnline/ EU\%20Kids\%20III/Reports/Intheirownwords020213.pdf 
Lobe, Bojana; Livingstone, Sonia; Ólafsson, Kjartan; Vodeb, Hana (2011). Cross-national comparison of risks and safety on the internet: Initial analysis from the EU kids online survey of European children. EU Kids Online, Deliverable D6. EU Kids Online Network, London, UK.

http://eprints.Ise.ac.uk/39608/1/Cross-national\%20 comparison\%20of\%20risks\%20and\%20safety\%20on\%20 the\%20internet(Isero).pdf

Martínez-Pastor, Esther; Sendín-Gutiérrez, José-Carlos; García-Jiménez, Antonio (2013). "Percepción de los riesgos en la red por los adolescentes en España: usos problemáticos y formas de control". Análisi: Quaderns de comunicació i cultura, n. 48, pp. 111-130.

http://www.raco.cat/index.php/Analisi/article/view/304861/394669

Ochaita, Esperanza; Espinosa, María-Ángeles; Gutiérrez, Héctor (2011). "Las necesidades adolescentes y las nuevas tecnologías de la información y la comunicación". Revista de estudios de juventud, n. 92, pp. 87-110.

http://www.injuve.es/sites/default/files/RJ92-07.pdf

Ólafsson, Kjartan; Livingstone, Sonia; Haddon, Leslie (2014). Children's use of online technologies in Europe. A review of the European evidence base. Revised edition. LSE, London: EU Kids Online.

http://www.dependencias.pt/ficheiros/conteudos/files/ Children.pdf

Padilla, Sonia; Rodríguez, Elisa; Álvarez, Míriam; Torres, Alezandra; Suárez, Arminda; Rodrigo, María-José (2015) "The influence of the family educational scenario on internet use of primary and secondary school children". Journal for the study of education and development, v. 38, n. 2; pp. 402-434. https://doi.org/10.1080/02103702.2015.1016749

Pelling, Emma L.; White, Katherine M. (2009). “The theory of planned behavior applied to young people's use of social networking web sites". Cyberpsychology \& behavior, v. 12, n. 6, pp. 755-759.

https://doi.org/10.1089/cpb.2009.0109

Rial-Boubeta, Antonio; Golpe-Ferreiro, Sandra; GómezSalgado, Patricia; Barreiro-Couto, Carmen (2014). "Variables asociadas al uso problemático de internet entre adolescentes". Health and addictions, v. 15, n. 1, pp. 25-38. http://www.redalyc.org/articulo.oa? id $=83938758003$

Ruggiero, Thomas E. (2000). "Uses and gratifications theory in the $21^{\text {th }}$ century". Mass communication \& society, v. $3, \mathrm{n}$. 1, pp. 3-37.

https://doi.org/10.1207/S15327825MCS0301_02

Rodríguez-García, Lorena; Magdalena-Benedito, José-Rafael (2016). "Perspectiva de los jóvenes sobre seguridad y privacidad en las redes sociales". Icono 14, v. 14, n. 1, pp. 24-49. https://doi.org/10.7195/ri14.v14i1.885

Rubio-Gil, Ángeles (2010). "Generación digital: patrones de consumo de internet, cultura juvenil y cambio social". Revista de estudios de juventud, n. 88, pp. 201-221.
http://www.injuve.es/sites/default/files/RJ88-14.pdf

Sabater-Fernández, Carmen (2014). "La vida privada en la sociedad digital. La exposición pública de los jóvenes en internet". Aposta, n. 61, pp. 1-32.

http://apostadigital.com/revistav3/hemeroteca/csabater.pdf

Sabater-Fernández, Carmen; López-Hernáez, Lara (2015). "Risk factors in cyberbullying. Frequency and exposition of personal data on the internet". International journal of sociology of education, v. 4, n. 1, pp. 1-25.

https://doi.org/10.4471/rise.2015.01

Sánchez-Martínez, Mercedes; Otero-Puime, Ángel (2010). "Usos de internet y factores asociados en adolescentes de la Comunidad de Madrid". Atención primaria, v. 42, n. 2, pp. 79-85.

https://doi.org/10.1016/j.aprim.2009.05.004

Sendín-Gutiérrez, José-Carlos; Gaona-Pisonero, Carmen; García-Jiménez, Antonio (2014). "Nuevos medios: usos comunicativos de los adolescentes. Perspectivas desde los nativos digitales". Estudios sobre el mensaje periodístico, v. 20, n. 1 , pp. 265- 280.

https://doi.org/10.5209/rev_ESMP.2014.v20.n1.45231

Sengupta, Anirban; Chaudhuri, Anoshua (2011). "Are social networking sites a source of online harassment for teens? Evidence from survey data". Children and youth services review, v. 33, n. 2, pp. 284-290.

http://online.sfsu.edu/anoshua/research/Sengupta_ Chaudhuri_WP.pdf

Slonje, Robert; Smith, Peter K. (2008). "Cyberbullying: Another main type of bullying?". Scandinavian journal of psychology, v. 49, n. 2, pp. 147-154. https://doi.org/10.1111/j.1467-9450.2007.00611.x

Staksrud, Elisabeth; Ólafsson, Kjartan; Livingstone, Sonia (2013). "Does the use of social networking sites increase children's risk of harm?". Computers in human behavior, v. 29, n. 1, pp. 40-50.

https://doi.org/10.1016/j.chb.2012.05.026

Utz, Sonja; Tanis, Martin; Vermeulen, Ivar (2012). "It is all about being popular: The effects of need for popularity on social network site use". Cyberpsychology, behavior, and social networking, v. 15, n. 1, pp. 37-42.

https://doi.org/10.1089/cyber.2010.0651

Valkenburg, Patti M.; Soeters, Karen E. (2008). “Children's positive and negative experiences with the internet. An exploratory survey". Communication research, v. 28, n. 5, pp. 652-675.

https://doi.org/10.1177/009365001028005004

Walrave, Michel; Heirman, Wannes (2013). "Adolescents, online marketing and privacy: Predicting adolescents' willingness to disclose personal information for marketing purposes". Children \& society, v. 27, n. 6, pp. 434-447. https://doi.org/10.1111/j.1099-0860.2011.00423.x 